\title{
A Gastrointestinal Stromal Tumor of the Jejunum Mimicking a Pelvic Mass in a Patient With Von Recklinghausen
}

\author{
Mehmet Yildirim, ${ }^{\mathrm{a},}$, Ozgur Oztekin ${ }^{\mathrm{b}}$, Nuket Eliyatkin ${ }^{\mathrm{c}}$, Hakan Postaci ${ }^{\mathrm{c}}$
}

\begin{abstract}
Von Recklinghausen's disease, is an autosomal dominant disorder with clinically characterized by gastrointestinal stromal tumors (GIST). We report a case of gastrointestinal stromal tumor of the jejunum masquerading as a pelvic mass in a patient with Von Recklinghausen's disease. A 43-year-old male complains of intermittent intestinal obstruction and radiological investigations detected a round shape pelvic solid lesion of approximately $5 \mathrm{~cm}$ in diameter. At laparatomy, a jejunum tumor was found in the pelvic localization and tumor resection was performed. Pathologic examination was revealed GIST.

In patients with von Recklinghausen's disease who complain of intestinal obstruction, a high suspicion degree of GIST is required.
\end{abstract}

Keywords: Von recklinghausen; Gastrointestinal stromal tumor

\section{Introduction}

Gastrointestinal stromal tumors (GISTs) are rarely seen

Manuscript accepted for publication April 19, 2012

${ }^{\mathrm{a}}$ Departments of Surgery, Izmir Bozyaka Teaching and Research Hospital, Izmir, Turkey

${ }^{\mathrm{b}}$ Departments of Radiology Izmir Bozyaka Teaching and Research Hospital, Izmir, Turkey

${ }^{c}$ Departments of Pathology, Izmir Bozyaka Teaching and Research Hospital, Izmir, Turkey

dCorresponding author: Mehmet Yildirim, Atakent Mah. Bergama 2 Apt. Giris:32 Daire:1,Bostanli, Izmir, Turkey.

Email: mehmetyildi@gmail.com

doi: $10.4021 /$ jes $40 \mathrm{w}$ mesenchymal tumors of the gastrointestinal tract that arise from the interstitial cells of Cajal. An association between the development of GIST and Von Recklinghausen's disease (VRD) has been established but interlink in this setting have not been elucidated [1]. GISTs are slow-growing tumors and a wide clinical spectrum ranging from benign incidental nodules to acute abdominal symptoms [2]. There are a few reports of jejunal GIST and Von Recklinghausen's disease association in the literature[3]. Therefore,we present the clinical and radiological findings, which mimicking a pelvic mass preoperatively, of a patient with jejunal GIST which was definite diagnosis confirmed following surgery.

\section{Case Report}

A 43-year-old man affected by VRD was admitted to our Department in December 2009 with a presumptive diagnosis of a pelvic tumoral mass affected the intestinal tract.

His medical history started three month ago when he was admitted to internal medicine clinic because of abdominal pain and distension. The patient had been a transabdominal pelvic ultrasonography and reported a well-bounded, heterogeneous mass measuring up to $5 \times 5 \mathrm{~cm}$ in the pelvic. It was located anterolateral to the rectum which displacing the rectum to left side. Our physical examination revealed multiple cafe-au-lait spots involving face and trunk, cutaneous neurofibromas on the trunk (Fig. 1A) and palpabl round shape mass on the lower abdominal quadrant. Ophthalmological examination was negative for Lisch's nodules. Laboratory routine tests as well as tumor markers (carcinoembryonic antigen, CA 19.9, CA125, AFP) were within normal values except ALT, GGT and ALP. Contrast enhanced CT of the pelvis identified a repressed segment of the rectum left laterally by a $6 \times 4.5 \mathrm{~cm}$ tumoral mass arising from the distal jejunal loops. The tumor was well demarcated with containing heterogeneous areas. According to the imaging studies, the pelvic mass was thought to be a serosal or submocosal originated tumor of the small intestine (Fig. 1B). Multiple masses were seen in CT scan of the liver at presentation. They showed rounded, hypodense enhancement in the right (segment 7 and 8) and left lobe (segment 2 and 4). 


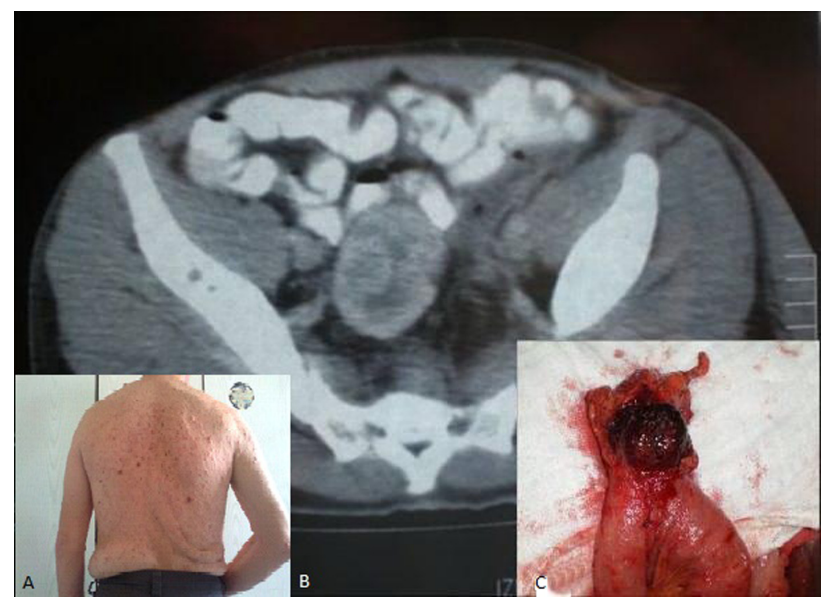

Figure 1. Neurofibromas and café-au-lait spots affecting the trunk (A). Axial CT examination of the pelvis showed an ovoid, exophytic inhomogeneously mass showing necrosis and haemorrhage, arising from the small bowel $(B)$. The resected segment of the jejunum shows haemorrhagic solid mass measuring up to $5.3 \times 3.5 \times 3.5 \mathrm{~cm}(\mathrm{C})$.

These masses were an average size of $3 \mathrm{~cm}$ with largest mass measuring $5 \mathrm{~cm}$ in the segment 7 . PET scan of the liver was showed hypermetabolic activity in all lesions. According to patient's history, it is considered that masses may be metastatic lesions. A fine needle biopsy of liver was showed no evidence of metastatic disease but fibrosis and necrotic liver cells. Colonoscopic examination was found normally.

At laparotomy, the patient was found to have an encapsuled, tender mass on located $40 \mathrm{~cm}$ distance from the Treitz ligament at the jejunum, which ranged from 5 to $4 \mathrm{~cm}$ in diameter, and adhering to serosal surface of the adjacent intestinal loops and rectosigmoid at the presacral region of the pelvis. The patient underwent resection of the bleeding tumor with involved segment of the jejunum. In macroscopic examination, the tumoral mass was measured $5.3 \times 3.5 \times 3.5$

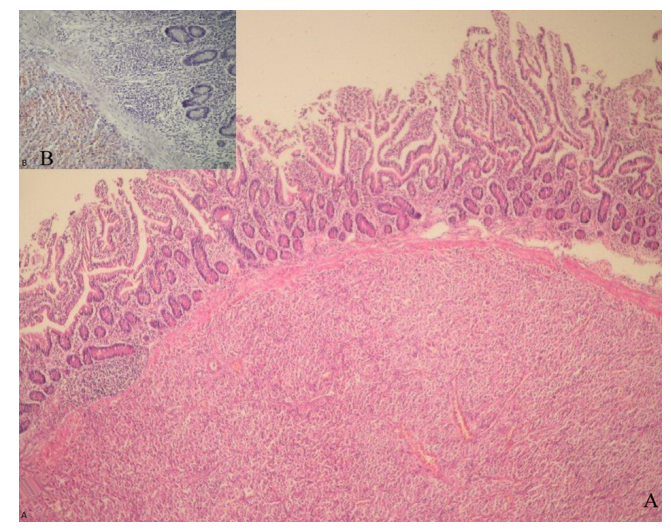

Figure 2. Histopathologic appearence of the tumor cells were showed submucosal growth of the tumor, leaving the surface epithelium intact (100 x, HE) (A). Immunohistochemical staining for CD117 showing strongly expressed in a diffuse pattern $(200 x)(B)$. $\mathrm{cm}$, well-defined, showing haemorrhagic appearance (Fig. 1B). Histopathologic examination of the specimen disclosed a spindle cell neoplasm transmurally infiltrating the whole small intestine wall. The tumor was composed of a diffuse proliferation of spindle cells and admixed of clear with giant cells in some areas. Peripheric circumferencial margins of the tumor were found no free in some areas. Immunohistochemically tumor cells expressed CD117 (50\%, ++), CD34 $(+)$ and vimentin $(+)$ in a diffused manner but no expressed desmin, S-100 protein, small muscle actin, musle-specific actin, CD10, CK7 and sitokeratin. The mitotic index was $1 / 50$ high-power fields. The tumor was reported as a lowrisk GIST (Fig. 2A-B).

The postoperative course was uneventful. The patient was discharged 5 days after operation. The patient was treat-

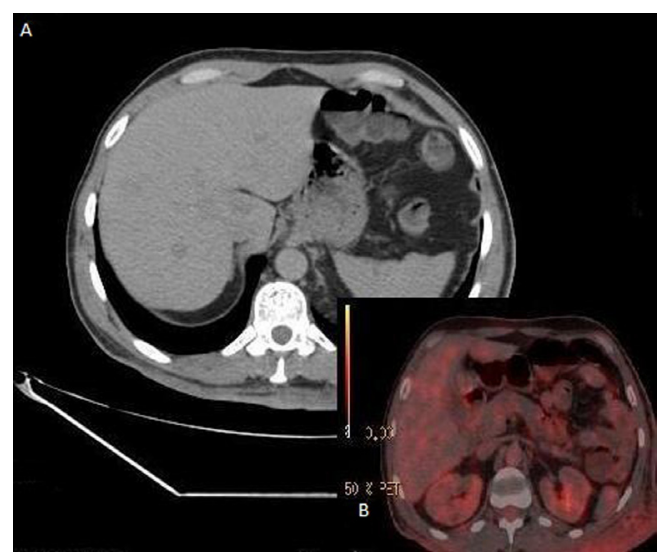

Figure 3. CT scan of the liver was showed visible regression of the lesion at the segment 8 and disappearance of the other lesions (A). PET scan was showed no hypermetabolic activity in all the masses on the 5-month follow up period (B). 
ed with adjuvant imatinib mesylate (Gleevec, Novartis) at $400 \mathrm{mg} /$ day. PET-CT scan of the liver was showed visible regression of the lesion at the segment 8 and no hypermetabolic activity in all the other masses on the 5-month follow up period (Fig. 3A-B). In the 18 months of the therapy, no relapses or evidence of metastatic diseases were demonstrated radiologically (CT and PET) or clinically.

\section{Discussion}

VRD is one of the most common genetic, neurogenic disease affecting cell types and the skin, bone, optic, endocrine, gastrointestinal (GI) and vascular systems [4]. It is a progressive disease, with variable clinical manifestations and associated with an increased incidence of neuroendocrine tumors, somatostinomas and non-neurogenic malignancies such as GISTs. Mutations at the NF1 gene and in the c-kit leading to increased expression of KIT that may predispose to development of a variety of tumors [5]. GISTs can occur more common in adults between fifth and sixth decades. Approximately $20-30 \%$ of GISTs occur in the small intestine.

In our patient, VRD was described with multiple cafeau-lait spots and generalized cutaneous neurofibromas in the face and trunk. A preoperative diagnosis of GIST is difficult given the abdominal pain and bowel habitus as in our case. The most common symptoms are abdominal pain, GI bleeding, luminal obstruction and abdominal mass. According to the studies $20 \%$ of patients are asymptomatic at the time of presentation [6]. In VRD, GIST of the jejunum is frequently non- specific/not-functioning and symptoms are related to local mass effects such as intestinal obstruction.

A total colonoscopy, CT, and MRI may help to precisely identify the suspected pelvic lesions preoperatively [7, 8]. Endoscopic US is a useful tool for evaluating luminal or extraluminal narrowing at the rectosigmoid, especially when the presenting symptoms not indicate a rectum cancer.

The characteristics of GISTs on CT and MRI are not specific, but can be suggestive to other GI malignancies[7]. On CT scan, typically evident of GISTs are intramural component, low attenuation areas suggesting hemorrhage, necrosis and air fluid levels (Torricelli-Bernouilli sign) in the mass. MRI studies show hypointense on $\mathrm{T}_{1}$-weighted images and hyperintense on T2-weighted images. In our case, CT was showed an ovoid well-marginated, an inhomogeneous mass with areas of necrosis extension into the rectum laterally, but no evidence of invasion to surrending tissues and the vessels. Gastric and rectal GISTs may lead to intraluminal component but this not detected in GISTs of the small intestine. Moreover the large tumoral mass of intestinal GISTs, which adhering to multipl bowel loops, makes it difficult to distinguish originating intestinal segment as in our case.

The differential diagnosis includes other mesenchymal tumors, leiomyomas, leiomyosarcomas, NETs, schwanno- mas and adenocarcinomas in the present of adenopathy, consantric luminal narrowing and large amount of ascites.

A fine-needle biopsy is appropriate to exclude other malignancies, but carries a risk of bleeding due to the friable nature of tumoral mass, and therefore is not suggested if surgery is planned.

In most cases, a clinic and pathologic diagnosis made after surgery with invasion of the adjacent organs and metastases of the distant organs [5]. Histologically there are no specific differences between GIST associated with VRD and those occurring in non-VRD patients. High grade GISTs are suggested by tumor size (the masses larger than $5 \mathrm{~cm}$. carries high grade malignancy risk than small masses $(<2 \mathrm{~cm}))$, and $>5$ mitoses/50 high-power fields [1]. In our case, mitotic index was suggested low-grade GIST but presence of adjacent organ adhesions, large tumor size and positive lateral margin considered high potentiel of malignancy and recurrence.

Complete surgical resection is the treatment of localized intestinal GISTs, and is associated with nearly 50-60\% 5-year survival rate. Enucleation may be inadequate since preoperatif diagnosis of malign potentiel is uncertain and also cause to peritoneal seeding. Imatinib mesylate (Gleevec ${ }^{\mathrm{TM}}$ ) is effective for adjuvant therapy in complately resected GISTs and also as the first-line therapy in patients with metastatic disease, with overall survival is over 4 years.

Liver masses respond to Gleevec therapy rapidly by showing reduction or disappearance of the lesions.Hypervasculer lesions may be missed in the single-phase CT study. For this reason,we identified the lesions pre and postoperatively with dual phase CT and PET scans in our case.

In conclusion, preoperatif diagnosis of a jejunal GIST located in the pelvis rarely possible in patients with VRD but imaging findings may be provide limited information if the mass originated from the bowel wall. In patients with von Recklinghausen's disease who complain of abdominal pain, intestinal obstruction, and abdominal mass a high suspicion degree of GIST is required.

\section{Conflict of Interest}

The authors have no potential conflicts of interest.

\section{Grant Support}

The authors have no financial support.

\section{References}

1. Miettinen M, Fetsch JF, Sobin LH, Lasota J. Gastrointestinal stromal tumors in patients with neurofibromatosis 1: a clinicopathologic and molecular genetic study of 
45 cases. Am J Surg Pathol. 2006;30(1):90-96.

2. Fuller CE, Williams GT. Gastrointestinal manifestations of type 1 neurofibromatosis (von Recklinghausen's disease). Histopathology. 1991;19(1):1-11.

3. Gaba S, Aslam M, Iqbal A. A Jejunal Gastrointestinal Stromal Tumour: an unusual cause of massive acute gastrointestinal haemorrhage with emphasis on pre intervention MDCT. J Radiol Case Rep. 2009;3(5):21-24.

4. Riccardi VM. Von Recklinghausen neurofibromatosis. N Engl J Med. 1981;305(27):1617-1627.

5. Andersson J, Sihto H, Meis-Kindblom JM, Joensuu H, Nupponen N, Kindblom LG. NF1-associated gastro- intestinal stromal tumors have unique clinical, phenotypic, and genotypic characteristics. Am J Surg Pathol. 2005;29(9):1170-1176.

6. Sandrasegaran K, Rajesh A, Rushing DA, Rydberg J, Akisik FM, Henley JD. Gastrointestinal stromal tumors: CT and MRI findings. Eur Radiol. 2005;15(7):14071414.

7. Savli M, Jamar B. Adenocarcinoma of the small bowel. Radiol Oncol. 2007; 41(2): 86-9.

8. Sofic A, Beslic S, Kocijancic I, Sehovic N. Ct colonography in detection of colorectal carcinoma. Radiol Oncol. 2010; 44(1): 19-23. 